DNA synthesis wheress the Fl fraction from the tumour cells seems to be free of this protein. Other more indirect evidence, for the association of a lysinerich histone with DNA synthesis, is provided by Yarbro (Biochim. Biophys. Acta, 145, 531; 1967). Hydroxyurea given to mouse ascites cells reduces DNA synthesis by 80 per cent while synthesis of RNA and non-histone protein is hardly affected. Total histone synthesis is reduced by about 35 per cent, but the very lysine-rich histone is reduced by 60 per cent. So Yarbro suggests there is an intimate association between DNA synthesis and the synthesis of the very lysinerich histone.

The arginine-rich histones are considered to be characteristic of stable, non-dividing cells by Evans and Rogers (Exp. Mol. Pathol., n, 105; 1967). They studied some of the histones from the tumour induced by the Shope papilloma virus. The tumour contains an arginase, and the histones, not surprisingly, contain a reduced arginine level. This has the effect of raising the relative level of lysine in the histone, and it is tempting to think that this leads to the onset of unregulated division and tumour initiation. Can the transition from division to differentiation really be mediated by the lysine-arginine balance?

\section{Recognition and Conformation}

\section{from our Molecular Biology Correspondent}

Some striking examples have been described which uphold the dogma of protein chemistry that the aminoacid sequence of a chain uniquely determines its conformation, and show in addition that the association of different chains may be required if the information contained in their sequence is to be expressed. The first example follows on one of the recent achievements of synthetic peptide chemistry, the total synthesis of insulin, reported simultaneously by Katsoyannis and his co-workers and by a group working in China. Although it has long been established that correct internal disulphide pairings are preferentially formed. in relation to incorrect ones when a single chain is allowed to refold from the reduced unfolded state, insulin, which has two chains joined by two of its three disulphide bonds, for a long time eluded attempts at correct recombination. Until the successful syntheses reported last year it was indeed often surmised that proper refolding and correct disulphide pairing could not occur independently of biosynthesis of the chains. This is now known not to be the case, and further details of recombination experiments have just been reported by Katsoyannis et al. (Biochemistry, 6, 2642; 1967).

If all three disulphide bonds of insulin are broken and allowed to reform, several intra-chain and interchain combinations will clearly be feasible. In practice, under defined conditions, recombination of $A$ and $B$ chains gives rise to only six products, which have been separated by ion-exchange chromatography. Two of these have the composition of $A$ chains, two others that of $B$ chains. It is not reported whether these contain inter- or intra-chain bonds, but it is in any event clear that only a small number of the possible pairings are sterically favoured. The remaining two components are both hormonally active and one is indistinguishable from native insulin. The nature of the other is unknown, and its proportion is determined by the precise conditions of recombination. It can. however, be converted into its native isomer by precipitation with picric acid. Katsoyannis et al. (ibid., 2656) have also prepared hybrid insulins from chains of different species, natural or synthetic, and fully active products have been obtained in vields ranging up to 50 per cent.

An important case of specificity of association between polypeptide chains occurs in antibodies. Immunoglobulins contain four chains, which are of two types, known as heavy $(H)$ and light $(L)$. Hybrid immunoglobulin molecules can be prepared, but it has been shown by Mannik that there is preferential recombination of $H$ and $L$ chains originating from the same molecular species. The antibody activity of the homologous species is also higher than that of the hybrids. Dorrington, Zarlengo and Tanford (Proc. US Nat. Acad. Sci., 58, 996; 1967) have now found physical evidence of a specific conformational interaction between homologous chains by the use of optical rotatory dispersion. The separated $H$ and $L$ chains each show a single Cotton effect maximum at 220 $230 \mathrm{~m} \mu$, but the intact immunoglobulin-as previously noted by Steiner and Lowey-shows a pattern in which the rotation is smaller, and at least three component Cotton effects can be discerned. Dorrington et al. find that this curve is completely regained when $H$ and $L$ chains from myeloma immunoglobulin are recombined. It is striking that recombination of heterologous chains - as in the case of a normal heterogeneous non-specific immunoglobulin-generates only a part of the pristine pattern, a minimum at about $240 \mathrm{~m} \mu$ being lost. Thus $H$ and $L$ chains will recognize each other and combine in specific manner, but a further interaction occurs only in the homologous case. It should be noted that the Cotton effects are very small, and not of a kind to suggest the presence or involvement of, for example, $\alpha$-helical structure. The conformational change could be confined to a very few residues, or such an effect could arise from large perturbation of a few chromophores, for example, by juxtaposition with charged groups.

Yet another instance of a recognition process between two chains leading to specific refolding is described by Taniuchi, Anfinsen and Sodja (ibid., 1235), who find that a staphylococcal nuclease of 149 residues can be cleaved by trypsin in the presence of inhibitor and calcium ions into three fragments of 5,44 and 100 residues. The two large fragments can then recombine non-covalently, when 8 per cent of the enzyme activity is regained. The system therefore recalls the subtilisinmodified ribonuclease of Richards, in which two fragments recombine to give substantially the activity of the native enzyme. The present case is particularly interesting because the structure is stabilized despite the absence of disulphide cross-links in this protein.

\section{Chain Termination}

\section{from our Cell Biology Correspondent}

LAST year Adams and Capecchi discovered that the initiation of protein synthesis in $E$. coli requires the insertion of $\mathrm{N}$-formylmethionine as the first aminoterminal amino-acid of the polypeptide chain. Since then, Capecchi has been working on the more intractable problem of how the synthesis of a polypeptide 